\title{
Dynamic surface tension of complex fluid-fluid interfaces A useful concept, or not?
}

\section{Journal Article}

Author(s):

Sagis, L. M. C.

Publication date:

2013-05

Permanent link:

https://doi.org/10.3929/ethz-b-000069454

Rights / license:

In Copyright - Non-Commercial Use Permitted

Originally published in:

The European Physical Journal Special Topics 222(1), https://doi.org/10.1140/epjst/e2013-01824-1 


\title{
Dynamic surface tension of complex fluid-fluid interfaces: A useful concept, or not?
}

\author{
L.M.C. Sagis ${ }^{1,2, a}$ \\ 1 Food Physics Group, Wageningen University, Bomenweg 2, 6703 HD Wageningen, \\ The Netherlands \\ 2 Polymer Physics, Department of Materials, ETH Zurich, Wolfgang-Pauli-Str. 10, \\ 8093 Zürich, Switzerland
}

Received 16 April 2013 / Received in final form 23 April 2013

Published online 17 June 2013

\begin{abstract}
Dilatational moduli are typically determined by subjecting interfaces to oscillatory area deformations, and are often defined in terms of the difference between the dynamic or transient surface tension of the interface (the surface tension in its deformed state), and the surface tension of the interface in its non-deformed state. Here we will discuss the usefulness of the dynamic surface tension concept in the characterization of dilatational properties of complex fluid-fluid interfaces. Complex fluid-fluid interfaces are interfaces stabilized by components which form mesophases (two-dimensionional gels, glasses, or (liquid) crystalline phases), as a result of in-plane interactions between the components. We will show that for such interfaces dilatational properties are not exclusively determined by the exchange of surface active components between interface and adjoining bulk phases, but also by in-plane viscoelastic stresses. The separation of these contributions remains a challenging problem which remains to be solved.
\end{abstract}

\section{Introduction}

The dilatational modulus of an interface is basically the two-dimensional equivalent of the bulk modulus defined for three-dimensional bulk phases. Its formal definition is (see [1] and references therein):

$$
E_{d} \equiv A\left(\frac{\partial \gamma}{\partial A}\right)
$$

where $E_{d}$ denotes the dilatational modulus, $A$ is the surface area of the interface, and $\gamma$ its surface tension. The latter is a function of the temperature and composition of the interface,

$$
\gamma \equiv \gamma\left(T^{s}, \rho^{s}, \omega_{(1)}^{s}, \ldots, \omega_{(N-1)}^{s}\right)
$$

\footnotetext{
${ }^{a}$ e-mail: leonard.sagis@wur.nl
} 
where $T^{s}$ is the surface temperature, $\rho^{s}$ is the overall surface mass density, and $\omega_{(A)}^{s}$ is the surface mass fraction of component $A$ in the interface.

Experimentally, the modulus is determined by subjecting an interface to sinusoidal area changes, and measuring the surface tension of the deformed interface $[1,2]$. In Oscillating Bubble Methods (OBM) this is done by analyzing the profile of the droplet, in Bubble Pressure Tensiometry (BPT) the pressure in the interior of the droplet is measured [2]. The profile or pressure data are then analyzed using the Laplace-Young equation. In Langmuir trough experiments the surface tension in the deformed state is measured directly, using a Wilhelmy plate [3].

In all these experiments the dilatational modulus is calculated from the transient surface tension data (after a Fourier analysis) using

$$
E_{d}=A \frac{\Delta \gamma}{\Delta A}
$$

where

$$
\Delta \gamma=\gamma\left(T^{s}, \rho^{s \prime}, \omega_{(1)}^{s \prime}, \ldots, \omega_{(N-1)}^{s \prime}\right)-\gamma_{\mathrm{nd}}\left(T^{s}, \rho^{s}, \omega_{(1)}^{s}, \ldots, \omega_{(N-1)}^{s}\right) .
$$

Here $\gamma_{\mathrm{nd}}$ is the surface tension of the non-deformed interface. The first term on the right hand side of this expression, is the surface tension of the deformed interface, and is often referred to as the dynamic or transient surface tension. The primes on the arguments in this term are used to signify that the adsorbed mass and composition of the interface may have changed as a result of the deformation.

For low molecular weight surfactants that exhibit negligible in-plane interactions after adsorption, this transient surface tension is mainly determined by the rate of exchange of surfactant between the interface and the adjoining bulk phases. Data are then typically analyzed using the Lucassen van den Tempel model $[4,5]$ or variations thereof. In fluid-fluid interfaces stabilized by colloidal particles, polymers, proteins, or protein aggregates, in-plane interactions can result in the formation of a complex microstructure, such as a two-dimensional gel, a glass phase, or (liquid) crystalline phase. When these interfaces are deformed in for example an OBM experiment, the dynamic surface tension determined from an analysis of the droplet profile may contain additional deviatoric stresses, and no longer has the characteristics of an isotropic tension. In this comment we discuss the usefulness of the dynamic surface tension concept for this type of interface, and discuss strategies on how to extract meaningful dilatational parameters for these interfaces using OBM, BPT, or Langmuir trough experiments.

\section{Dynamic surface tension of complex fluid-fluid interfaces}

In OBM and BPT experiments the dynamic surface tension is determined from the Young-Laplace equation, given by

$$
P^{(2)}-P^{(2)}=2 \gamma H
$$

where $P^{(2)}$ is the pressure in the interior of the droplet, $P^{(1)}$ is the pressure in the outer phase, and $H$ is the mean curvature of the interface. Elsewhere in this issue [6], we have seen that for complex fluid-fluid interfaces we need to use a generalized form of this equation to analyze profile or pressure data, referred to as the jump or surface momentum balance [1,7-12]. In [6] we have discussed conditions for which this generalized balance reduces to the Young-Laplace equation (5). Let us assume that an OBM or BPT experiment is performed in such a way that in-plane inertial 
stresses, and viscous stresses exerted on the interface by the adjoining bulk phases are negligible. We will also assume that contributions from the bending rigidity of the interface to the momentum balance are negligible. Moreover, we assume the deformation is small and uniform. Under these conditions the momentum balance of the interface reduces to $[6]$

$$
P^{(2)}-P^{(1)}=2 \gamma H+2 H \operatorname{tr}\left(\boldsymbol{\sigma}^{s}\right)
$$

where $\operatorname{tr}\left(\boldsymbol{\sigma}^{s}\right)$ is the trace of the surface extra stress tensor, $\boldsymbol{\sigma}^{s}$. We see that for a complex fluid-fluid interface, the pressure difference over the interface is determined not only by an isotropic surface tension, but also by in-plane viscoelastic stresses (possibly anisotropic). Lumping these two contributions into a single isotropic dynamic surface tension is inconvenient, since it obscures the true nature of the response of the interface.

Another argument against the introduction of a dynamic surface tension is given by nonequilibrium thermodynamics. Savin et al. [13] recently analyzed the local equilibrium assumption for interfaces, in the context of gauge invariance (any description of a multiphase system should be independent of the choice of the exact location of the Gibbs dividing surface, and should hence be invariant under small displacements of this surface). The local equilibrium assumption states that for a system not in global equilibrium (i.e. intensive variables such as temperature and density vary in time and space), at any point in the system, one can assume thermodynamic equilibrium in a small neighborhood around this point. The analysis by Savin et al. [13] (and studies cited therein) support the validity of this assumption also for interfaces. This implies that the surface tension of an interface not in equilibrium (i.e. the first term on the right hand side of (4)) is given by the same thermodynamic expression as the equilibrium surface tension, and the difference between the two is merely due to a difference in the state variables $T^{s}, \rho^{s}$, and $\omega_{(A)}^{s}$, induced by the deformation of the interface. So there is no need to introduce the concept of a dynamic surface tension, we just need to realize that the surface tension $\gamma$ appearing in equation (6) is a function of time.

\section{Strategies for determining dilatational properties of complex interfaces: Limiting cases}

In this section a number of experimental protocols are suggested to obtain dilatational properties for complex fluid-fluid interfaces, using either OBM, BPT or a Langmuir trough. Let us assume we have an interface where surface tension, deviatoric stresses, and bending rigidity may all contribute to the response of the interface to an applied deformation. The latter contribution will in most cases be negligible, but there are some exceptions, such as vesicle membranes, or interfaces in phase separated biopolymer mixtures, where these contributions may be significant $[1,14-20]$. In [6] we saw that for such a system the pressure difference over the interface is given by

$$
P^{(2)}-P^{(1)}=2 \gamma H-k C_{0} K+2 H \operatorname{tr}\left(\boldsymbol{\sigma}^{s}\right)
$$

where $k$ is the bending rigidity of the interface, $C_{0}$ is its spontaneous curvature, and $K$ is the Gaussian curvature of the interface (equal to $1 / R^{2}$ for a spherical drop with radius $R$ ). The analysis of the pressure data will greatly simplify if one of the contributions on the right hand side of (7) is dominant in the response. To establish this we need to determine the dependence of the response on droplet size, amplitude of oscillation, and frequency of oscillation. 
Let us first look at response of the interface to variations of the droplet size. Let us assume we are performing an experiment using an OBM device, and we use its standard image analysis mode, which extracts an effective "surface tension" by solving the Young-Laplace equation (we use quotes here since the extracted parameter need not be an actual tension, but could also be a stress). In this approach the terms on the right hand side of (7) all are lumped in this effective surface tension, which takes the form

$$
\begin{aligned}
\gamma_{\mathrm{eff}} & =\gamma-k C_{0} \frac{K}{2 H}+\operatorname{tr}\left(\boldsymbol{\sigma}^{s}\right) \\
& =\gamma-k C_{0} \frac{1}{R}+\operatorname{tr}\left(\boldsymbol{\sigma}^{s}\right) .
\end{aligned}
$$

The scaling of the terms on the right hand side of (8) with droplet radius $R$, are respectively, $\gamma \sim R^{0}, k C_{0} / R \sim R^{-1}$, and $\operatorname{tr}\left(\boldsymbol{\sigma}^{s}\right) \sim R^{-1}$. If in this OBM experiment we plot the effective surface tension, measured at various droplet sizes, against $1 / R$, and we obtain a horizontal line, then we know the surface tension term must be dominant in the response. If we obtain a linear curve with a negative slope, the response is bending rigidity dominated, and when the curve is linear with a positive slope, the response is dominated by deviatoric stresses.

We could have a situation where the last two terms in (8) are both large but cancel each other, leading to an effectively surface tension dominated response. To check for this the experiment can be repeated at various deformation amplitudes $\delta R$. The first two terms on the right hand side of (8) are independent of $\delta R$, whereas the last term is linear in $\delta R$. So when $\gamma_{\text {eff }}$ is linearly dependent on strain (for small amplitudes) we know the response is stress dominated. Strain sweeps not only help us to identify whether deviatoric stresses contribute to the response of the interface to a deformation. When these stresses are dominant, strain sweeps can also provide information about the nature of the interface. The structure of the interface may give rise to strain thinning or strain thickening behavior, and the occurrence of these phenomena can provide a clue what the structure of the interface is (although usually we will need additional information from surface shear or structural characterization methods to confirm this).

To summarize, if the effective surface tension of the interface is independent of $\delta R$ and $1 / R$, we have a surface tension dominated response, when the response is independent of $\delta R$ and proportional to $1 / R$ we have a bending rigidity dominated response, and when we have a response proportional to $\delta R / R$ the response is dominated by deviatoric stresses (Table 1).

The properties we determine in each of these cases are different. When the response of the interface is surface tension dominated, we find that $\gamma_{\text {eff }}(t)=\gamma(t)$. So in this case the deformation of the interface changes the surface concentrations, and the magnitude of the dilatational properties is determined by the rate of exchange of the surface active components between interface and the adjoining bulk phases. The modulus we determine in this case is the thermodynamic dilatational modulus

$$
E_{d 0}=\frac{\gamma_{\mathrm{eff}}-\gamma_{\mathrm{nd}}}{d \ln A}=\frac{\gamma-\gamma_{\mathrm{nd}}}{d \ln A}
$$

When the response is rigidity dominated, and the deformation does not alter the surface tension significantly, we find that $\gamma_{\mathrm{eff}}(t)=\gamma_{\mathrm{nd}}(t)-k C_{0} / R(t)$. And hence in this case the relevant property we extract from the determination of $\gamma_{\text {eff }}$ is not a dilatational modulus, but the rigidity term $k C_{0}$ :

$$
k C_{0}=\left(\gamma_{\text {eff }}-\gamma_{\text {nd }}\right) R .
$$


Table 1. Overview of typical responses of fluid-fluid interfaces to an applied dilatational deformation.

\begin{tabular}{llll}
\hline $\begin{array}{l}\text { Dominant } \\
\text { contribution }\end{array}$ & $\begin{array}{l}\text { Surface } \\
\text { tension }\end{array}$ & $\begin{array}{l}\text { Deviatoric } \\
\text { stresses }\end{array}$ & $\begin{array}{c}\text { Bending } \\
\text { rigidity }\end{array}$ \\
\hline Scaling of $\gamma_{\text {eff }}(t)$ with $R$ & $R^{0}$ & $R^{-1}$ & $R^{-1}$ \\
& & & $(\delta R)^{0}$ \\
Scaling of $\gamma_{\text {eff }}(t)$ with $\delta R$ & $(\delta R)^{0}$ & $\delta R$ & $\omega^{0}$ \\
& & & \\
Scaling of $\gamma_{\text {eff }}(t)$ with $\omega$ & $\left.\omega^{1 / 2} a\right)$ & $\left.E_{d}^{\prime} \sim \omega^{2}, E_{d}^{\prime \prime} \sim \omega^{1}, \omega<<1 / \tau_{d} b\right)$ & \\
& & $\left.E_{d}^{\prime} \sim \omega^{0}, E_{d}^{\prime \prime} \sim \omega^{-1}, \omega>>1 / \tau_{d} b\right)$ & \\
& & $E_{d}^{\prime} \sim \omega^{0}, E_{d}^{\prime \prime}=0^{c)}$ \\
& & $E_{d}^{\prime} \sim \omega^{n}, E_{d}^{\prime \prime} \sim \omega^{m}, m, n=0.1-0.2$ & \\
& & $E_{d}+G_{s}$ & $k C_{0}$ \\
$\begin{array}{l}\text { Relevant measured } \\
\text { parameter }\end{array}$ & $E_{d 0}$ & & \\
\hline
\end{tabular}

a) Lucassen van den Tempel model [4,5].

b) Linear viscoelastic interface, described by the surface Maxwell model.

c) Linear purely elastic interface.

d) Interfaces stabilized by a $2 \mathrm{~d}$ glass phase, close to the glass transition.

When the response is dominated by deviatoric stresses, and the surface tension does not change significantly during deformation, we have $\gamma_{\text {eff }}(t)=\gamma_{\text {nd }}+\operatorname{tr}\left[\boldsymbol{\sigma}^{s}(t)\right]$. An example of a system behaving in this way would be an interface stabilized by a mixture of colloidal particles, and a low molecular weight surfactant, in which the colloidal particles aggregate into a two-dimensional particle gel, and the low molecular weight surfactant occupies the "pores" in this structure. When deformations are slow, and the exchange of the surfactant with the bulk phase is sufficiently fast such that during deformation the surface tension in the pores of the two-dimensional particle gel does not change, then the response of the interface will be dominated by the resistance of the particle gel structure against deformation. The property we determine in this case depends on the characteristics of the interface. For a purely elastic interface in the linear response regime, we have $\operatorname{tr}\left(\boldsymbol{\sigma}^{s}\right)=\left(E_{d}+G_{s}\right) d \ln A$, so then we find

$$
E_{d}+G_{s}=\frac{\gamma_{\mathrm{eff}}-\gamma_{\mathrm{nd}}}{d \ln A}
$$

where $E_{d}$ and $G_{s}$ are respectively the dynamic dilatational and surface shear modulus. For large deformations, outside the linear response regime, we would obtain the apparent dynamic moduli of the interface. The fact that there is a dependence on shear properties in this response means that for a proper characterization of the dilatational properties of such an interface, we need to also perform surface shear experiments to determine $G_{s}$.

Apart from varying droplet radius and deformation amplitude, we can also obtain information on the nature of an interface by performing frequency sweeps. When we have an interface where the response to a deformation is dominated by surface tension changes, then the dilatational modulus is usually described well by the Lucassen van den Tempel model $[4,5]$ (or variations thereof), and the scaling of the dilatational storage modulus $E_{d 0}^{\prime}$ with frequency $\omega$ is given by $E_{d 0}^{\prime} \sim \sqrt{\omega}$. If however we have an interface where the response of the interface to a deformation is dominated by deviatoric stresses, and the stress deformation behavior of the interface is described well by a linear viscoelastic model, such as the surface Maxwell model, then the dynamic dilatational storage modulus $E_{d}^{\prime}$ and dynamic dilatational loss modulus $E_{d}^{\prime \prime}$ scale with 
frequency as $[1]$

$$
E_{d}^{\prime}=E_{d 0}+\frac{\varepsilon_{d} \omega^{2} \tau_{d}}{1+\omega^{2} \tau_{d}^{2}} \quad E_{d}^{\prime \prime}=\frac{\varepsilon_{d} \omega}{1+\omega^{2} \tau_{d}^{2}}
$$

where $\varepsilon_{d}$ is the surface dilatational viscosity, and $\tau_{d}$ is the dilatational relaxation time of the interface. From these expressions we see that at low frequencies $\left(\omega<<1 / \tau_{d}\right)$, these two moduli scale with frequency as

$$
E_{d}^{\prime} \sim \omega^{2} \quad E_{d}^{\prime \prime} \sim \omega .
$$

For high frequencies $\left(\omega>>1 / \tau_{d}\right)$, they scale with frequency as

$$
E_{d}^{\prime} \sim \omega^{0} \quad E_{d}^{\prime \prime} \sim \omega^{-1}
$$

Interfaces with an almost purely elastic stress-deformation behavior will have a scaling behavior with frequency given by $E_{d}^{\prime} \sim \omega^{0}$ and $E_{d}^{\prime \prime} \approx 0$. Interfaces stabilized by components forming a soft two-dimensional glass phase, tend to show a scaling given by $E_{d}^{\prime} \sim \omega^{n}$ and $E_{d}^{\prime \prime} \sim \omega^{m}$, with $n$ and $m$ of the order of $0.1-0.2$ (close to the glass transition) [21].

We see that when one of the contributions to $\gamma_{\text {eff }}$ is dominant, we can determine that dominant contribution by performing droplet size variations, strain sweeps, and frequency sweeps. Once the dominant contribution is identified, we can extract meaningful either thermodynamic or dynamic properties of the interface. But how do we analyze systems where more than one contribution contributes significantly to the response of the interface. We will discuss this situation in the next section.

\section{Interfaces with mixed behavior}

Let us assume we have an interface in which as a result of an applied deformation the surface tension of the interface changes, and we induce deviatoric stresses in the interface. So equation (8) reduces to

$$
\gamma_{\mathrm{eff}}(t)=\gamma(t)+\operatorname{tr}\left[\boldsymbol{\sigma}^{s}(t)\right]
$$

This implies that both the thermodynamic and dynamic dilatational modulus (and possibly also the dynamic surface shear modulus) are contributing to the response of the interface. If both these contributions are significant over the entire range of accessible droplet sizes, frequencies, and strain sweeps, the separation of these contributions is a tedious problem, which in general cannot be tackled with OBM or other dilatational methods alone.

If we can establish the composition of the interface as a function of time (during the process of deformation), and from equilibrium experiments we have established the equation of state of the interface, then we can calculate $\gamma(t)$ in (15). With $\gamma(t)$ known, we can subsequently extract the dynamic dilatational modulus from the stress contribution in (15) (if $G_{s}$ is either known or negligibly small). This would amount to doing a two-dimensional rheo-optics experiment, that is, simultaneously resolving the rheological response and structural evolution of an interface. For interfaces rheooptics is still a field in development [22], and few methods are currently available for structure evaluation during deformation.

$2 \mathrm{D}$ rheo-optics experiments have been performed on interfaces stabilized by colloidal particles with diameters above $1 \mu \mathrm{m}$, using microscopy [23-27], but for these systems the response tends to be dominated by the deviatoric stresses, and other contributions tend to be negligible. These experiments are most often performed in 
surface shear mode, and mainly serve to investigate the nonlinearities that occur at high deformation rates, and link these to structural rearrangements induced by the applied deformation.

Smaller particles in the nanometer range could be analyzed with techniques such as scanning angle reflectometry [28,29], but this technique has mainly been applied to solid-liquid interfaces, and not on fluid-fluid interfaces subjected to a deformation. It would only be applicable in Langmuir trough experiments, since in OBM and BPT methods the location of the interface is not fixed. But even in a Langmuir trough the optical analysis would be complicated by small waves induced by the barrier motion.

Protein, polymer, or lipid stabilized interfaces could be studied by neutron or Xray reflectivity measurements, but again only on flat interfaces. Although for these systems structural analysis has been performed for interfaces at rest [30-35], experiments under flow have so far scarcely been performed. An example of such an experiment is the one designed by Wagemaker et al. [36], who used neutron reflectivity to examine interfaces expanded in an overflowing cylinder.

When experimental data on structural evolution is hard to obtain, mesoscopic simulations of the structural evolution may provide an interesting alternative to examine surface rheological data. We would then need to develop mesoscopic models for the dynamics of interfaces stabilized by, for example, 2D entangled polymer gels, or 2D liquid crystalline phases. Mesoscopic nonequilibrium thermodynamics (see the discussions by Lervik and Kjelstrup [37], and Santamaria-Holek et al. [38] elsewhere in this issue), may prove to be a valuable tool to construct such models. But we must conclude that also this field is still in development. For now the extraction of reliable values for surface properties of complex interfaces, where multiple effects contribute to the response of the interface to a deformation, remains an arduous task.

\section{Conclusions and outlook}

In view of the arguments presented in this paper, the dynamic surface tension is not a useful concept for complex fluid-fluid interfaces, mainly because it obscures the true nature of the response of an interface to a deformation. Apart from changes in surface tension, deformations may induce deviatoric stresses in complex interfaces, and the bending rigidity of the interface may also contribute to the response of an interface. We have shown here that a protocol involving droplet size variations, strain amplitude sweeps, and frequency sweeps can be used to identify the dominant contributions to the response of an interface, and when only one of these is dominant in a given range of $R, \delta R$, and $\omega$, reliable values for the surface properties (either $E_{d 0}, E_{d}$, or $k C_{0}$ ) can be obtained. When more than one contribution is dominant the separation of these contributions can in general not be done with dilatational experiments alone. A combination with surface shear, structural analysis methods, and mesoscopic simulations may then be needed to unravel the dynamics of the interface. Particularly in the fields of structural analysis during deformation, and mesoscopic surface simulations significant progress is needed for this to come within reach.

\section{References}

1. L.M.C. Sagis, Rev. Mod. Phys. 83, 1367 (2011)

2. A. Javadi, N. Mucic, M. Karbaschi, J. Won, M. Lotfi, A. Dan, V. Ulaganathan, G. Gochev, A. Makievski, V. Kovalchuk, et al., Eur. Phys. J. Special Topics 222, 7 (2013)

3. L. Wilhelmy, Ann. Phys. 119, 177 (1863) 
4. J. Lucassen, M. van den Tempel, Chem. Eng. Sci. 27, 1283 (1972)

5. J. Lucassen, M. van den Tempel, J. Colloid Interface Sci. 41, 491 (1972)

6. L.M.C. Sagis, Eur. Phys. J. Special Topics 222, 31 (2013)

7. L.E. Scriven, Chem. Eng. Sci. 12, 98 (1960)

8. J.C. Slattery, Chem. Eng. Sci. 19, 379 (1964)

9. B.J.A. Zielinska, D. Bedeaux, Physica A 112, 265 (1982)

10. D.A. Edwards, H. Brenner, D.T. Wasan, Interfacial Transport Phenomena and Rheology (Butterworth-Henemann, Boston, 1991)

11. R. Gatignol, R. Prud'homme, Mechanic and Thermodynamic Modeling of Fluid Interfaces (World Scientific Publishing, Singapore, 2001)

12. J.C. Slattery, L.M.C. Sagis, E.S. Oh, Interfacial Transport Phenomena, 2nd edn. (Springer, New York, 2007)

13. T. Savin, K.S. Glavatskiy, S. Kjelstrup, H.C. Öttinger, D. Bedeaux, Eur. Phys. Lett. 97, $40002(2012)$

14. J.B.A.F. Smeulders, C. Blom, J. Mellema, Phys. Rev. A. 42, 3483 (1990)

15. J.B.A.F. Smeulders, C. Blom, J. Mellema, Phys. Rev. A. 46, 7708 (1992)

16. K.H. de Haas, G.J. Ruiter, J. Mellema, Phys. Rev. E 52, 1891 (1995)

17. E. Scholten, L.M.C. Sagis, E. van der Linden, J. Phys. Chem. B 108, 12164 (2004)

18. E. Scholten, L.M.C. Sagis, E. van der Linden, Macromolecules 38, 3515 (2005)

19. E. Scholten, L.M.C. Sagis, E. van der Linden, J. Phys. Chem. B 110, 3250 (2006)

20. L.M.C. Sagis, J. Controlled Release 131, 5 (2008)

21. P. Cicuta, E.J. Stancik, G.G. Fuller, Phys. Rev. Lett. 90, 236101 (2003)

22. E. van der Linden, L.M.C. Sagis, P. Venema, Curr. Opinion Colloid Interface Sci. 8, 349 (2003)

23. H. Hoekstra, J. Vermant, J. Mewis, Langmuir 19, 9134 (2003)

24. S. Reynaert, P. Moldenaers, J. Vermant, Phys. Chem. Chem. Phys. 9, 6463 (2007)

25. M.G. Basavaraj, G.G. Fuller, J. Fransaer, J. Vermant, Langmuir 22, 6605 (2006)

26. K. Masschaele, J. Fransaer, J. Vermant, J. Rheol. 53, 1437 (2009)

27. B. Madivala, J. Fransaer, J. Vermant, Langmuir 25, 2718 (2009)

28. E.A. van der Zeeuw, L.M.C. Sagis, G.J.M. Koper, Macromolecules 29, 801 (1996)

29. E.A. van der Zeeuw, L.M.C. Sagis, G.J.M. Koper, E.K. Mann, M.T. Haarmans, D. Bedeaux, J. Chem. Phys. 105, 1646 (1996)

30. L.T. Lee, E.K. Mann, O. Guiselin, D. Langevin, B. Farnoux, J. Penfold, Macromol. 26, 7046 (1993)

31. E. Dickinson, D.S. Horne, R.M. Richardson, Food Hydrocolloids 7, 497 (1993)

32. P.J. Atkinson, E. Dickinson, D.S. Horne, R.M. Richardson, J. Chem. Soc., Faraday Trans. 91, 2847 (1995)

33. G. Harzallah, V. Aguie-Beghin, R. Douillard, L. Bosio, Int. J. Biol. Macromol. 23, 73 (1998)

34. S.W. An, R.K. Thomas, F.L. Baines, N.C. Billingham, S. Armes, J. Penfold, Macromol. 31, 7877 (1998)

35. V.M. Kaganer, H. Möhwald, P. Dutta, Rev. Mod. Phys. 71, 779 (1999)

36. M. Wagemaker, F.J.G. Boerboom, H.J. Bos, A.A. van Well, Physica B 283, 278 (2000)

37. A. Lervik, S. Kjelstrup, Eur. Phys. J. Special Topics 222, 143 (2013)

38. I. Santamaria-Holek, Z.J. Grzywna, J.M. Rubi, Eur. Phys. J. Special Topics 222, 129 (2013) 